\title{
Surface topologies of thin aluminum films and absorbing properties of metal dielectric structures in the microwave range
}

\author{
Ibraim Shevketovich Fitaev, Vulf Borisrvich Orlenson, Yuri Viktorovich Romanets, \\ and Alim Seit-Ametovich Mazinov". \\ V.I. Vernadsky Crimean Federal University, 295007, Simferopol, Russia
}

\begin{abstract}
The paper presents experimental and theoretical results on the surface topography of thin aluminum films. The ambiguous topology dynamics of the thin-film structure is represented as a function of the increase in the bulk mass of the deposited material, which leads to an occasional rise in roughness reaching its maximum at a film thickness of 7 $\mathrm{nm}$. At same time on the same thickness of the conductive layer the maximum of the absorption coefficient variability is observed. A theoretical analysis of the optical coefficients depending on the size of the conductive film substantiated the existence of extremes, but at smaller thicknesses.
\end{abstract}

\section{Introduction}

The development of the microelectronic industry along with the use of semiconductor materials and dielectrics triggered the need for thin conductive films used as data buses, termination pads, screens, etc. [1]. As is shown in [2], conductive films with a thickness of a few nanometers can absorb almost half of the incident EMF (electromagnetic field) power in the frequency range from the very few to several hundred gigahertz [3, 4]. Yet, for largescale industrial production, it is necessary to use cheap dielectric substrates, which have complicated surface relief. Therefore, it is of particular relevance to search for consistent patterns of conductive layers formation for MDS structures used as shielding coatings against microwave radiation in the wavelength range from 7 to $15 \mathrm{~cm}$ and to study the relationship between their structure relief and EMF absorption.

\section{Thin film synthesis}

Aluminum was chosen as a conductive material, since it is a frequently used material in the microelectronic industry [5]. The MDS structures were obtained by the magnetron sputtering system URM 3.1279.0129 with the use of 99,98 pure aluminum target. Additional ion cleaning and substrate planetary rotation system ensures uniform coating. The changeable speed of the substrate rotation enabled us to alter the material deposition characteristics.

*Corresponding author: mazinovas@cfuv.ru 
The glass square (rectangular) plates were used as substrates. The substrates type choice was made taking into account that the sizes of most objects requiring shielding have areas exceeding a number of square meters. It is very difficult to produce a uniform conductive surface with height deviation less than a few nanometers, not to mention the industrial conditions for large areas coating, where it is almost impossible. Therefore, the film deposition on $\sim 10$ nanometer inhomogeneous relief that is very close to industrial production led to the choice of cheap glass substrates for samples being tested in interaction with an electromagnetic field [6]. It seemed logical to continue the research by choosing the coating method where the vacuum part performed two-stage pumping out, and also the diffusion pump system provided the residual vacuum level of $10^{-5}$ Torr.

Visualization of the surface structure was carried out in several stages. The coating uniformity general assessment was carried out by the MCCO U4.2 type long-focus microscope with further analysis in white light on the MII-4M. The geometry detalization of the specific sections was carried out using the MII-4M monochromat interference lines on a $0.53 \mu \mathrm{m}$ semiconductor laser. A more detailed lateral and vertical, about $50 \mathrm{~nm}$ and more than $2 \mathrm{~nm}$ respectively resolution was obtained by the SP-NT-MDT NANOEDUCATOR II. The final stage in the surface morphology study was atomic force scanning using SPM NT-MDT INTEGRA. The atomic force scanning of the substrate surface made it possible to investigate the basic relief in more detail and showed that most of the defects had a base size dimensions of $2 \times 2 \mu \mathrm{m}$, with an average height range $Z_{d}=20 \pm 5 \mathrm{~nm}$. The analysis of the surface revealed the presence of relatively high, but rather rare peaks.

\section{The absorbing properties of films and their relation to the surface topography}

Microwave research was carried out by closed waveguide space of $34 \times 72 \mathrm{~mm}$ in the frequency range from 2 to $4.3 \mathrm{GHz}$ using the Panorama P4226 vector network analyzer, from Mikran (Fig. 1). In this case, the traveling wave mode was provided by a matched load and directional couplers for the transmitted and reflected components of the test radiation. The $15 \times 15 \mathrm{~mm}$ ( $10 \%$ waveguide cross-section area) samples were chosen to avoid short line effect that can occur due to the waveguide and surface film AC connection.

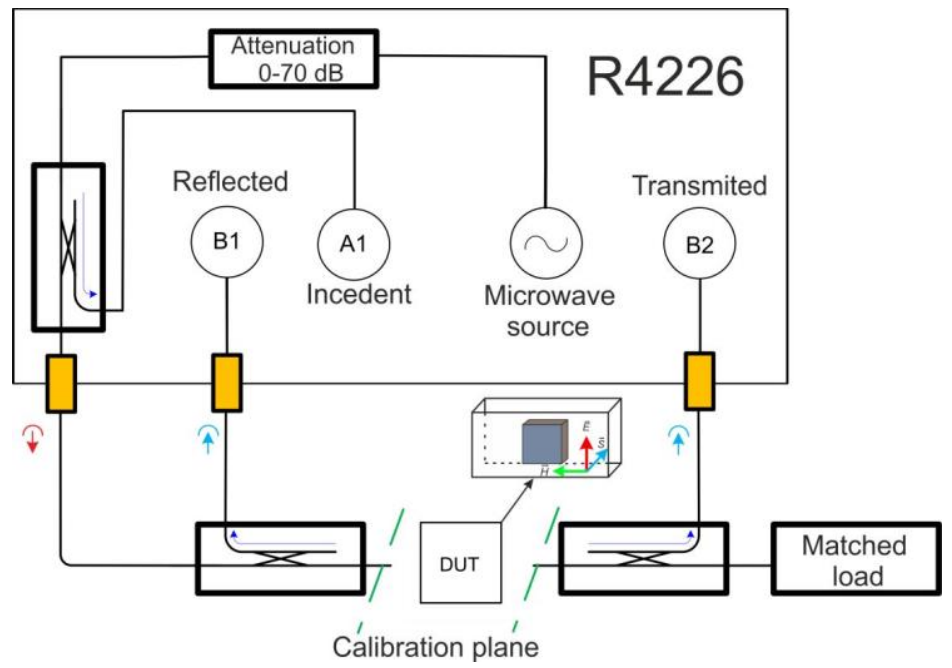

Fig. 1. Block diagram of the measuring system. 
The samples were fixed at the waveguide cross-section by a dielectric substrate made of transparent electromagnetic material. Thus, the sample was placed at the maximum of the electric field component $[4,7]$.

Vector network analyzer makes it possible to get the S-parameters of the measured device. The reflection, transmission and absorption coefficients can be obtained from Sparameters by squaring the module of the desired parameter:

$$
R=\left|S_{11}\right|^{2} ; T=\left|S_{21}\right|^{2} ; A=1-\left|S_{21}\right|^{2}-\left|S_{11}\right|^{2} \text {. }
$$

The thicker the film becomes the higher the absorption coefficient is, however, such dynamics is observed up to a certain thickness, while after reaching it the absorption starts to decrease. An absorption "peak" was detected at a $7 \mathrm{~nm}$ film thickness. The general optical coefficients correlation patterns were averaged over three sample batches from various technological cycles. Moreover, at the same time, the diagnostics of the general surface geometry was carried out for each batch, which was evaluated integrally by 2D AFM images and statistical data on the roughness and the surface heights distribution of the films (Fig. 2-3).

It was found that the film at the initial stage of its growth (up to $1 \mathrm{~nm}$ ) tends to monotonously decrease in average roughness to a thickness of almost $5 \mathrm{~nm}$, while the maximum height difference (up to $3 \mathrm{~nm}$ ) increases.
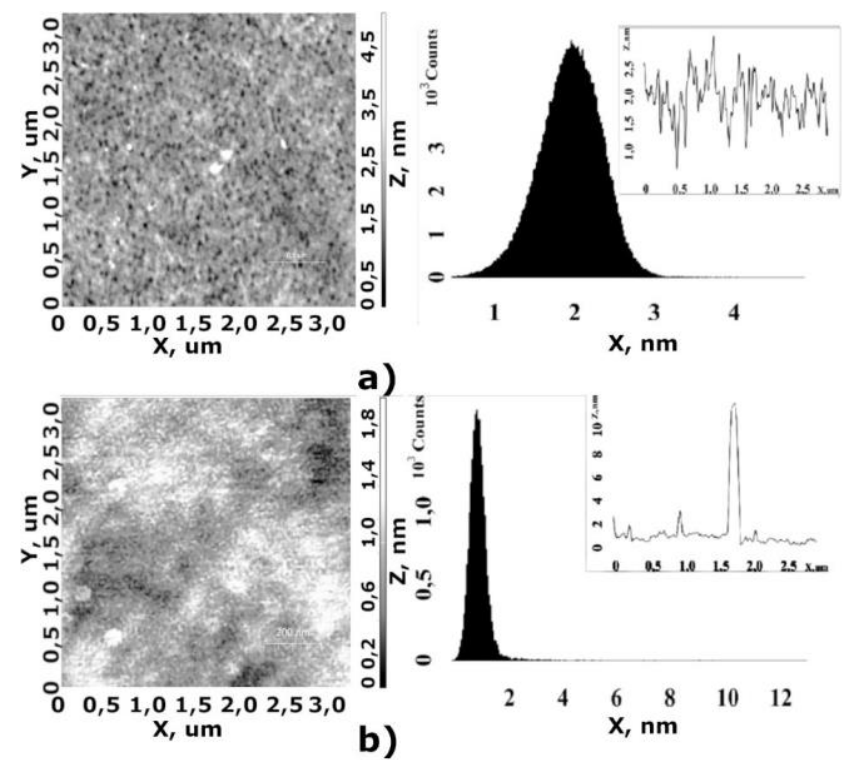

Fig. 2. Morphology, distribution of heights and revealed characteristic profilogram for: a) $3 \mathrm{~nm}$; b) $5 \mathrm{~nm}$.

The detected "peak" at $7 \mathrm{~nm}$, observed with the increase in film thicknesses, is a rare exception of the monotonic relief "smoothing", but such surface characteristic is still in accordance with thin metal film absorption study results (Fig. 4).

An analysis of the statistical data on the growth of films obtained using ACM and processing of the results allows to make the following assumption: the growth of an aluminum film on a glass substrate occurs in accordance with the Volmer-Weber mechanism [8], that is, during magnetron deposition, the formation of a continuous coating occurs in conformity with the islet-type growth mechanism.

Presumably the initial conductive particles formation occurs at the step areas, recess areas and also at the substrate stoichiometry atomic lattice defects. It can be explained by 
the fact that the deposited conductive substance atoms can travel a while across the substrate surface until their energy is used up or they are trapped by the strong bond substrate areas.
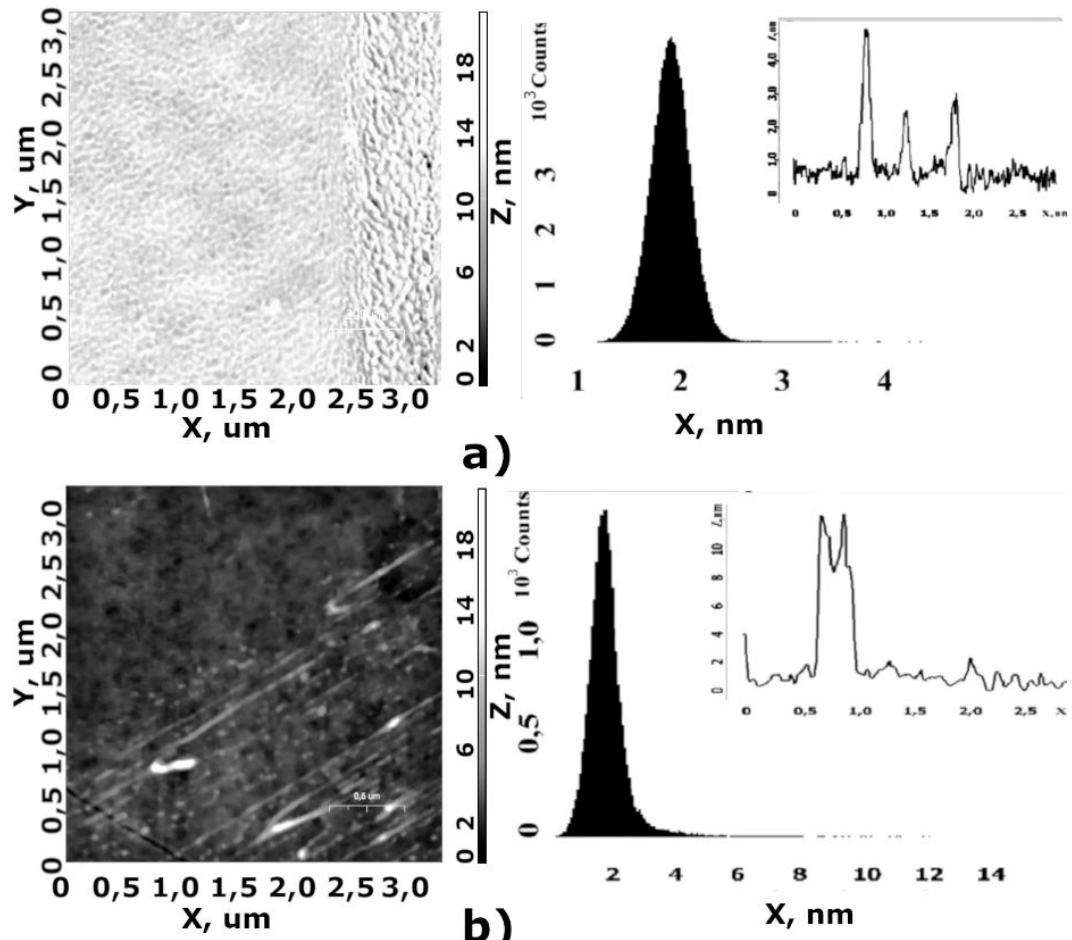

$\mathbf{X}, \mathbf{n m}$

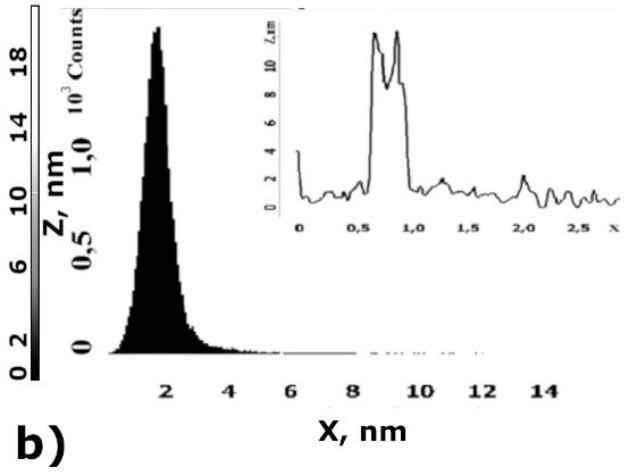

Fig. 3. Morphology, distribution of heights, revealed characteristic profilogram:

a) $7 \mathrm{~nm}$; b) $10 \mathrm{~nm}$.

As a result, such defects and surface irregularities can significantly increase the conductive particles genesis rate. In our case, there is an underlying surface with complex, diverse geometry, which is caused by various surface polishing and grinding imperfections at the final stage of the production process [9].

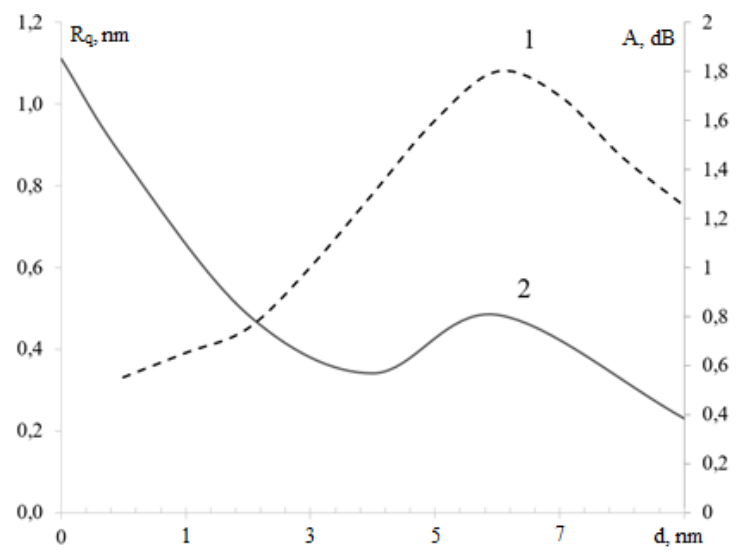

Fig. 4. Dependence on film thickness: 1 - attenuation coefficient; 2 - surface roughness. 
Therefore, at the initial period of time, the conductive particles are formed in the surface inhomogeneities. Further material deposition leads to particle growth and their transformation into large agglomerates of the condensed phase [10]. Assuming that lateral growth rate exceeds the vertical one, after a while the conductive islet structures merge and form variuos types of conductive channels and voids across the substrate surface, which were observed by the AFM scanning (Fig. 3a). Further deposition creates continuous metal film.

In areas with different elevations and peaks, the conductive material thickness is smaller than in areas at their footing and where there are recesses and plains. Evidently, there is still elastic tension between the thin metal regions and the new deposited film parts, which can be accounted for by the non-periodic substrate atomic lattice structure. Consequently, for the deposited atoms the more energetically favorable regions appear. In certain areas of already produced film this creates conditions for nanocrystalline structure selective growth and accounts for the extremes of roughness growth dynamics at the film thickness of $7 \mathrm{~nm}$ (Fig. 4). These nanocrystallites are larger than the original islets formed at the initial stage of the film growth. When the critical conditions of atomic lattices mismatch on the entire area of the sample are eliminated, the relief smoothing of an already continuous film with minimal roughness occurs, which is can be seen from the graph in Fig. 4 , at the thickness of $10 \mathrm{~nm}$.

The theoretical studies of plane wave scattering from thin metal film at normal incident can be done by model approximation of the MDS by a thin conductive region surrounded by two semi-infinite dielectric (free space) spaces. The optical coefficients dependences on the film thickness were determined by Fresnel - Airy model analytical expressions [11, 12]. Figure 4 shows the dependences of the optical coefficients on the film thickness in the microwave range. At specific conductivity of $\sigma=10^{6} \mathrm{~S} / \mathrm{m}$, a maximum absorption is achieved at the thickness of $d=3 \mathrm{~nm}$ (Fig. 5a), while at crystalline aluminum conductivity of $\sigma=3,8 \cdot 10^{7} \mathrm{~S} / \mathrm{m}$, the absorption maximum is achieved at $d=0,3 \mathrm{~mm}$ (Fig. 5b). The coincidental match of the experimental absorption results with fig.5a but not with fig. $5 \mathrm{~b}$ can be explained by the fact that, after sputtering, the deposited conductive material becomes amorphous aluminum, the conductivity of such aluminum being lower than the one of crystalline aluminum, and it is of the $\sim 10^{6} \mathrm{~S} / \mathrm{m}$ order.

a)

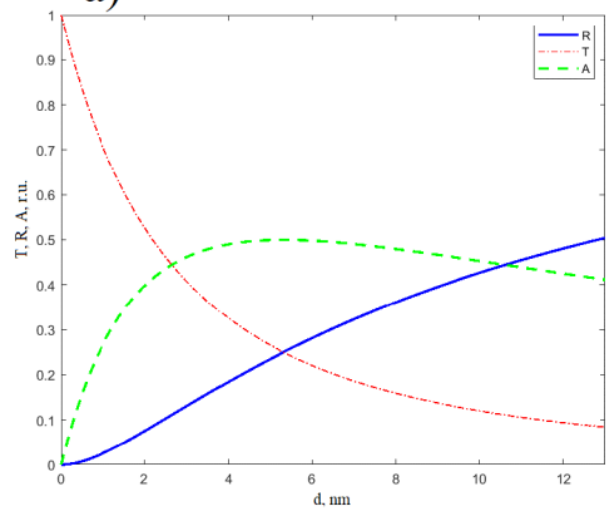

b)

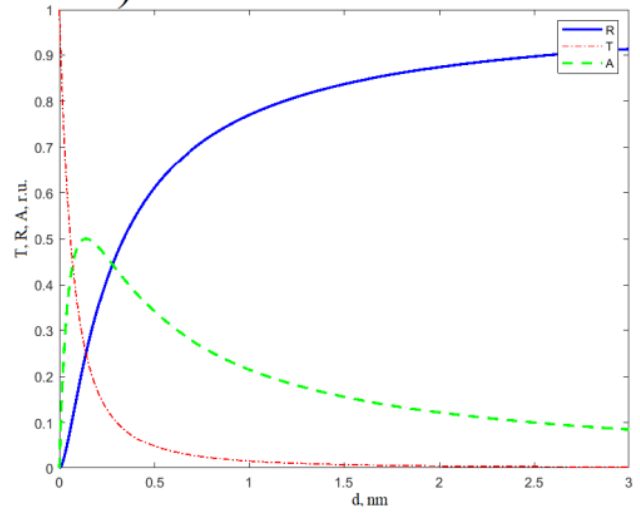

Fig. 5. Simulation results of optical coefficients dependence on the film thickness.

Such model assumes conductive layer smooth boundaries which is impossible to obtain in real thin films, created with a semi-industrial production method. This explains the inconsistency of the experimental measurements with the theoretical model calculation results. To understand the influence of the conductive layer inhomogeneity on the 3 
coefficients the rigorous coupled wave analysis (RCWA) [3] was used. Simulation of reflection and transmission based on RCWA made it possible to analyze the optical coefficients changes depending not only on the film thickness but also on the rate of substrate surface overlapping by the conductive material.

\section{Conclusion}

The article gives an outline of the aluminum film formation dynamics on glass substrate. It was observed that the substrate surface geometry determined the initial growth of conductive film individual sections, which further led to the increase in relief height differences of up to $25 \mathrm{~nm}$, while the average roughness monotonically decreased $(<1 \mathrm{~nm})$. The part of thickness of $5 \mathrm{~nm}$ and above is of particular interest as it is where the increase in roughness and height differences reoccurs, the maximum of the increase being observed at $7 \mathrm{~nm}$ thickness. The roughness maximum (at $7 \mathrm{~nm}$ film thickness) coincidentally match the microwave energy absorption maximum, which is also confirmed by model calculations where the absorption maximum depends on the thickness and the fill factor of the metallized coating.

\section{References}

1. D. Vogler, The Roadmap to 5nm: Convergence of Many Solutions Needed [online], SEMI, SEMI Foundation Continues. Available at: http://www.semi.org/en/node/55926 (10.04.2019)

2. N. Ahmad, J. Stokes, Neil A Fox, M. Teng, Cryan Martin James, Ultra-Thin Metal Films for Enhanced Solar Absorption. Nano Energy, 1(6) (2012)

3. G. Nimtz, U. Panten, Broad band electromagnetic wave absorbers designed with nanometal films. Ann. Phys. (Berlin)

4. Sucheng Li, Shahzad Anwar, Weixin Lu, Zhi Hong Hang, Bo Hou, Mingrong Shen, and Chin-Hua Wang. Microwave absorptions of ultrathin conductive films and designs of frequency-independent ultrathin absorbers. AIP ADVANCES, 4 (2014)

5. G. Bräuer, Magnetron Sputtering. Comprehensive Materials Processing. (2014)

6. S.I. Baskakov, Fundamentals of electrodynamics and radio wave propagation, 416 (2017)

7. Arsenichev S.P., Grigoryev E.V., Zuev S.A., Starostenko V.V., Taran E.P., Fitaev I.Sh., Electromagnetic waves and electronic systems, 22(2), pp. 48-53 (2017)

8. S. A. Kukushkin, A. V. Osipov, Advances in Physical Sciences, 168(10), 1083 (1998)

9. M. G. Moharam, Drew A. Pommet, Eric B. Grann, "Stable implementation of the rigorous coupled-wave analysis for sur-face-relief gratings: enhanced transmittance matrix approach. J. Opt. Soc. Am. A, 12(5), pp. 1077-1086 (1995)

10. R.C. Rumpf. Improved formulation of scattering matrices for semi-analytical method that is consistent with convention. Progress In Electromagnetics Research B, 35, pp. $241-261$ (2011)

11. G.V. Rosenberg Thin layer optics, 570 (1958)

12. M.M. Gorshkov, Ellipsometry (Sovetskoe Radio, Moscow, 1973) 